\title{
Aciclovir \& IVIG fail in CMV
}

The use of aciclovir prophylaxis with or without IV immune globulin (IVIG) has little if any, effect on the incidence of cytomegalovirus (CMV) infection in solid organ transplant recipients.

Dr Thomas C Bailey and colleagues from the Washington University School of Medicine, Saint Louis, US, came to this conclusion after evaluating these drugs as CMV prophylaxis in 21 seronegative patients receiving solid organ transplants from seropositive donors. They believe that aciclovir 'cannot be recommended' for CMV prophylaxis without an additional confirmatory study.

Aciclovir is not approved in the US for this indication, nor is there substantive data supporting such use. Despite this, high-dose aciclovir has been advocated as a more cost-effective and convenient alternative to the FDA approved treatment, CMV-hyperimmune globulin.

Dr Bailey and colleagues report that most patients in their trial received therapeutic ganciclovir despite the use of prophylaxis with oral aciclovir with or without IVIG. They also cite several reasons to be sceptical of previously published data on the efficacy of aciclovir as prophylaxis.

Aciclovir has little in vitro activity against CMV at concentrations achievable clinically, the clinical evidence is conflicting, and other investigators have also questioned the efficacy of aciclovir for this indication.

Bailey TC, Ettinger NA, Storch GA, Trulock EP, Hanto DW, et al. Failure of high-dose oral acyclovir with or without immune globulin to prevent primary cytomegalovinus disease in recipients of solid organ transplants. American Journal of Medicine 95: 273-278, Sep 1993 